\title{
$\mathfrak{Z} \mathfrak{u} \mathfrak{i} \mathfrak{c} \mathbf{t}$.
}

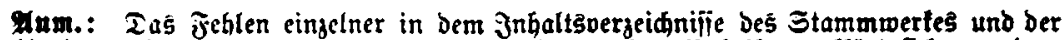

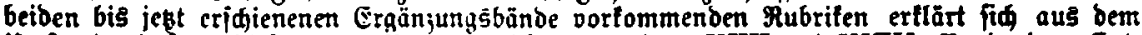
Umitanbe, baß gu diejen neue Meditsgrunojäze aus bem XVII. und XVIII. Bande ber "Ents fđeibungen" niḍt jul verzeidinter toarert.

\section{Erfte Dutrilung.}

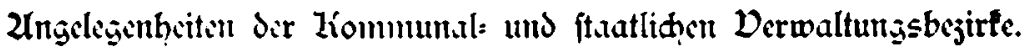

I. Brovinzialocrbände

seite

A. Mahlangcligacnhiti:: .

II. Arevie.

A. Bahlangelegentfei:en . .

2

B. F̂reişabgaben . . . . .

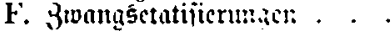

III. Etäbtc.

A. Etäbtiidge Gemeitrbeabynben

12

C. Berbältnifị Det itäbtiiden Sommunalbeanten

D. Etäbtijác \$allen.

IV. Auts̄berbände

V. Randgemeinden und (Butôbcjirte.

A. Bemeinbeabgahen und Dieatiti, Ganeinbcuermöget. . . . . 33

B. Tisziplinarangelegenticiten ber Bemeindebeamten . . . . . 39

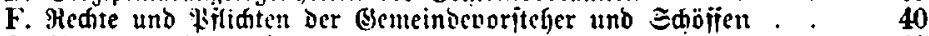

(i. Itmenngelegenhciter . . . . . . . . . . . . . . 40

II. Bemeindeljolzungen

\section{3urite Abteilung.}

Kíirde, Ethuli uno pariontuftand.

1X. 2ic Edulc.

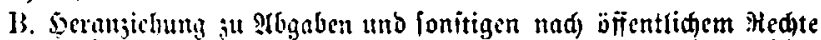

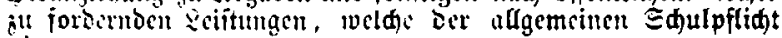
diener.

(. $\Xi d) u l b a u t e r$

XI. a Etaatzanacbörigfrit

\section{Dritte Alteilung.}

Polijitlithi 2lngivlesinhiten.

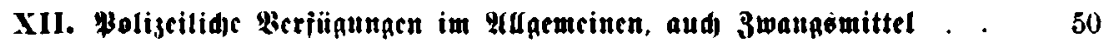
XIII. 2ie 3agd.

A. ingoidlinte

B. ingobesirfi uto ingoverpadiung

XV1. Das diafice 
XIX. Das Bewerbe. Eeite

A. Die Cafts unb ミdantwirtidaft . . . . . . . . . . . 63

E. Sebeammens unb Entbinbungsanjtalten . . . . . . . . 64

F. פergte unb Epotbeter . . . . . . . . . . . . . 64

H. Wandergewerbefinein . . . . . . . . . . . . . . . 65

I. Oewerblide \&nlagen . . . . . . . . . . . . . . 65

L. Innungen . . . . . . . . . . . . . . . . . . . 66

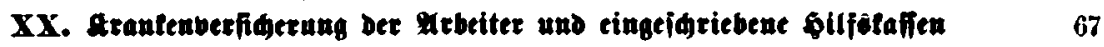

XXI. Da sauwefen . . . . . . . . . . . . . . . . . . . . 69

XXII. anfiedrugen und stolonten . . . . . . . . . . . . . . . 71

XXIII. Serficerungen . . . . . . . . . . . . . . . . . . . . 72

XXIV. Die Sandelofammern . . . . . . . . .

interte 2uteilung.

Der Derwaltungsprojé.

XXVI. Das Etreitberfahren . . . . . . . . . .

XXYII. Inftruttionelle \$erfügungen über das Etreitwerfahen . . .

CGronologijđes Megiiter . . . . . . . . . . . . . . . . .

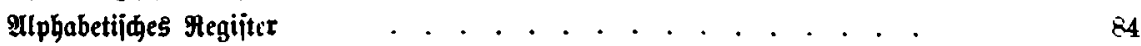

